\title{
Künstlicher Pneumothorax, angelegt im 4. Monate der Gravidität ${ }^{1}$ ).
}

\author{
Von
}

Dozent Dr. D. O. Kuthy, Budapest und Dr. G. Lobmayer, Budapest.

Die kurze Skizze des lehrreichen Falles lässt sich folgendermassen geben: Es erscheint in der Sprechstunde eine 19 jährige Frau, Gattin eines besser situierten Landmannes von der grossen Ebene, Mutter eines Kindes. Durch ihre Krankheit scheint sie fast nochmals so alt zu sein. Gegenüber ihrem Höchstgewicht (als erwachsenes Mädchen) besteht angeblich eine Abnahme von ca. $20 \mathrm{~kg}$, davon entfallen auf die letzten 2 Monate ca. $6 \mathrm{~kg}$. Seit einem viertel Jahr Husten, zweimal Hämoptyse; bei der ersten Konsultation besteht Subfebrilität. Appetit schlecht, reichliche Nachtschweisse. Die physikalische Untersuchung der Lungen ergibt verhältnismässig wenig Veränderung, die Röntgenoskopiebedeutend mehr, was im allgemeinen als ein böses Prognostikum gilt, weil es die disseminierte Lungentuberkulose charakterisiert.

Da die vorhandene spezifische Peribronchitis und insuläre Bronchopneumonie auf der rechten Seite viel mehr ausgesprochen ist, legen wir mit Hilfe der Brauerschen Schnittmethode einen rechtsseitigen Pneumothorax an. Der Erfolg ist vorläufig ein brillanter, das wir mit um so mehr Genugtuung konstatieren, da es sich inzwischen herausstellt, was die Frau bei ihrer Ausfrage verheimlichte, dass sie sich zur Zeit der ersten Konsultation bereits im 4. Monat der Gravidität befand. - Unsere Freude bezüglich des Erfolges dauerte jedoch nicht sehr lange. Post partum brach das ganze Resultat zusammen:

1) Vortrag, gehalten auf der I. Generalversammlung des Tuberkulosevereins ungarischer Ärzte zu Budapest, am 12. A pril 1913. 
es stellte sich in Begleitung von hohem Fieber von neuem eine grosse Entkräftung ein und 2 Monate nach der Entbindung ist die Kranke verstorben.

Epikrise. Die an einer bösartigen Lungentuberkulose leidende sravide Frau hat somit den künstlichen Preumothorax sehr gut vertragen. Ihr somatischer Zustand wurde durch die Kompressionstherapie - in Begleitung einer Körpergewichtszunahme, welche die durch den graviden Uterus bedingten Plus an Gewicht bedeutend iiberflügelte - namhaft gebessert, und im Lungenprozess, soweit dieser nach Anlegung eines Pneumothorax objektiv beurteilbar ist, trat Stillstand ein; post partum et puerperium ging jedoch alles z ug runde.

Dieser aus dem Falle resultierende praktische Schluss wïrde völlig mit der gangbaren, im allgemeinen gewiss richtigen Anschauung übereinstimmen, welche nicht nur der Entbindung, dem Kindbett und der Laktation, sondern auch der Gravidität an sich eine ungünstige Wirkung auf den tuberkulösen Lungenprozess zuschreibt.

Wenn wir es aber in Betracht ziehen, dass sich in der Literatur einzelne Anschauungen finden (wie z. B. die von Aufrecht in der jüngst erschienenen 2. Auflage seines Tuberkulosehandbuches), nach denen die Gravidität an und für sich nicht immer unginstig auf die Lungentuberkulose einwirkt, ja es könne der gravide Zustand - durch seinen Einfluss auf die Motilität der Lungen, auf die Zirkulation, durch Erzeugung einer in der zweiten Hälfte der Gravidität erhöhten Nahrungsaufnahme - sogar gelegentlich günstig für den Lungenprozess sein: so wäre es möglich, unsere Auffassung bezüglich des vorliegenden Falles derart zu ändern, dass wir annehmen, dass die junge Frau mit schwerer disseminierter Lungentuberkulose, welche eigentlich bereits doppelseitig war und die Pneumothoraxtherapie bloss als letzten Versuch zuliess, ihre fast bis zur Entbindung dauernde namhafte Besserung vielleicht teilweise eben $\mathrm{mit} H \mathrm{Hilfe} \mathrm{der}$ Gravidität erreicht hatte.

Damit soll freilich nicht gesagt werden, dass die Gravidität für schwindsüchtige Frauen vielleicht ein willkommener Zustand wäre (unser Standpunkt ist, wie erwähnt, ebenfalls der entgegengesetzte), nur soviel müssen wir zugeben, dass in unserem künstlichen Pneumothoraxfalle bei der Erzeugung des zwar transitorischen günstigen Erfolges der Gravidität eine gewisse Rolle nicht abzusprechen sei. Besonders weisen wir auf den Umstand hin, dass die konstant hohe Stellung des Zwerchfells, verursacht durch den bis zum VIII. Graviditätsmonat sich nach aufwärts drängendem Uterus, die immobilisierende und komprimierende Wirkung des Nitrogens auf die Lungen 
direkt steigern konnte. Hierauf deuten die unten angeführten Angaben der detaillierten Krankengeschichte des Falles, welche zeigen, dass die Wiederverschlimmerung eigentlich nicht nach der Entbindung, sondern schon vor derselben in der Endperiode der Gravidität eintrat, welche Erscheinung wir geneigt sind so zu erklären, dass der schwangere Uterus sub finem graviditatis eine Vorwärtssenkung erleidet, somit seine raumverengende Wirkung auf die Brustorgane weniger ausgesprochen wird.

Die detaillierte Beschreibung des Falles lautet folgendermassen:

Frau B. B., 19 Jahre alt, Mutter eines $1^{1 / 2}$ jährigen Kindes. Auf Anraten ihres Hausarztes wendet sie sich an einen von uns (Dr. Kuthy) am 27. Juni 1912.

A namnese. Brnder an Tuberkulose gestorben. Patientin selbst war früher nie ernstlich krank. Seit einem $1 / 4 \mathrm{Jabr}$ Husten, zweimal Hämoptyse. Das Körpergewicht war angeblich vor $2^{1 / 2}$ Jahren (vor ihrer Heirat) in Winterkleidung $76 \mathrm{~kg}$, vor $2 J a h r e n$ bloss ca. $60 \mathrm{~kg}$, vor 2 Monaten $58 \mathrm{~kg}$. Gegenwärtig fühlt sie sich schwächer, hat schlechten Appetit und Stuhlverstopfung, unruhigen Schlaf; hustet mittelmässig viel, angeblich ohne Auswurf; hat dyspnoetische Gefühle und starken Nachtschweiss, angeblich auch Fieber, Puls (sitzend) 120, Körpergewicht 52,6 kg. Patientin macht den Eindruck einer abgemagerten Person.

Objektiver Bef und. Thorax longus, Angulus Ludovici prominens, rechtsseitiges Akromialsymptom ${ }^{1}$ ) (auch die Schmerzen, welche die Patientin angibt, beziehen sich auf die rechte Thoraxhälfte). Aktive Mobilität der Lungenränder beiderseitig, besonders rechts eingeschränkt, doch auch hier nicht völlig aufgehoben. Der Luftschall des $K$ rö nigschen Feldes ist rechts verschwunden, links zentral eingeengt. Die leise Perkussion gibt rechts bis unter die Klavikula einen gedïmpften, links bloss supraklavikulär einen verkürzten Schall. Hinten ist die A pexgegend rechts gedämpft, links nur verkürzt; weiter unten lässt sich in der Mitte der interskapulären Region bei vorsichtigem Beklopfen beiderseitig ein verkülzter Perkussionsschall konstatieren. Ausgebreitetes, vesikobronchiales Atmen über beide Oberlappen, besonders rechts — ohne Rhonchi. Die von Dr. Kuthy darchgeführte Röntgendurchleuchtung stellte einen pathologischen Schatten der rechten Spitze, der rechten Hilusgegend und der ganzen rechtseitigen, paravertebralen Zone fest; weiter zeigte sich eine besonders von vorne gut hervortretende und vorwiegend am rechten Unterlappen sehr ausgesprochene Verstärkung des Bronchialbaumschattens (peribronchiale Infiltrate). Links wurde der Unterlappen am Röntgenschirm normal befundeu; in den sonstigen Teilen der linken Lunge waren ebenfalls pathologische Schatten, analog denen der rechten Seite, doch betleutend weniger stark, vorhanden, bloss ein Plus war hier röntgoskopisch zu finden, nämlıch bei ventrodorsaler Durchleuchtung ein auffallender Schattenstrang vom Hilus auf- und auswärts ziehend. Die von Kollege E. F is cher verfertigte Röntgenphotographie zeigte analoge Verhältnisse, nur mit grösserer Differenzierung der Details, mit stärkerem Hervortreten der zahlreichen zerstreaten bronchopneumonischen Herden besonders der rechten Lunge.

Anlegung des Pneumothorax nach Brauer am 27. Juni 1912 im Sanatorium Pajor. In die rechte Pleurahöhle werden $700 \mathrm{ccm} \mathrm{N}$ eingelassen.

1) Kuthy: Das Akromialsymptom bei Lungentuberkulose, Zeitschr. f. Tbk., Bd. XIV, H. 3, und: 'Transactions of the 6. internat. Congr. on Tuberculosis, Washington 1908. 
Nachher Euphorie; die Temperatur behält ibren subfebrilen Charakter (bis $37,4^{\circ}$ ) noch 2 Tage lang; am 1. Juli die ers te $\mathrm{N}$ a c h full $1 \mathrm{ung}$ (Insufflation von $1200 \mathrm{ccm} \mathrm{N}$ ), nach welcher sich Afebrilität einstellt (Max. 36,7 ${ }^{\circ}$. Ständiges Wohlbefinden. Dio auf die erste Nachfüllung folgende Durchleuchtung zeigte die rechte Lunge in die paravertebrale Zone kontrahiert und komprimiert, bloss die Spitze sitzt noch fest in der Pleurakuppel. Mit diesem Status reist die Kranke nach Hause.

Nach 2 Wochen stellt sie sich wieder vor. Sie gibt jetzt an, dass sie sich kräftiger fühlt, der Appetit gut, Stahl regelmässig, obzwar der Schlaf noch zeitweise unruhig ist; Husten weniger, Auswurf 0, Dyspnoe 0, Nachtschweiss 0 . Fieber konnte nicht konstatiert werden. Das Aussehen der Patientin ist gut, Puls 112, Gewicht: 54,3 kg (Zunahme: $1,7 \mathrm{~kg}$ ). Die Untersuchung stellt das Bostehen des Pneumothorax arteficialis fest mit einer gewissen Verschiebung der Gasblase nach hinten, indem sich die rechte Iunge vorne etwas mehr entfaltete, hinten jedoch stärker zusammengepresst wurde. Herzdislokation nach links ist vorhanden : die relative Herzdämpfung beginnt am linken Sternalrande. Die Auskultation ergibt bestehende, relative Subaktivität der rechten Lunge (am hinteren, unteren Margo einige atelektatische Krepitationen). Auf eine inzwischen schon dennoch eingetretene relative Abnahme des künstlich erzeugten intrathorakalen Druckes haben hingewiesen, dass bei tiefer Inspiration auch die rechte Hälfte des $\mathrm{Zwerch}$ fells sich auffallend nach abwärts bewegt und dass man das Atemgeräusch hinten unten auch über der rechten Thoraxhälfte hört.

Am Tage nach dieser Untersuchung (17. Juli) die z weite $\mathrm{Nachfüllung.}$ Insufflation von $1350 \mathrm{ccm} N$ (Schlussdrack \pm 0 ). Nach derselben geht die Patientin vom im III. Stockwerke befindlichen Operationssaal des Sanatoriums in ihre Wohnung im II. Stock auf eigenen Füssen und reist am nächsten Tage nach Hause. Auch nach dieser Einblasung totale Euphorie, bloss am selben Abend leichte Subfebrilität $\left(37,2^{\circ}\right)$. Am darauffolgenden Tage bereits wieder normale Temperaturen (unter $37,0^{\circ}$ ).

Die nach der zweiten Reinsufflation vollführte Untersuchung ergab folgendes : die relative Herzdämpfungsgrenze liegt ein Querfinger links vom linken Sternalrande, Spitzenstoss in der Paramamillarlinie (so benennt $\mathrm{Kuthy}$ eine vertikale Linie zwischen Linea mamillaris und Axillaris anterior); rechts vorne durchwegs - auch die Spitzengegend inbegriffen - Schachtelton und totales Fehlen von Atemgeräusch; rechts hinten paravertebral wenig Atmung (die Abnahme der Intensität der Atmung ist besonders unten sehr ausgesprochen). Die Röntgendurchleuchtung konstatiert den nunmehr kompleten Pneumothorax, da jetzt auch die Spitze (aus dem lateralen Teile der Pleurakuppel) verschoben ist und die ganze rechte Lunge sich in die Regio paravertebralis zurückzog. Ihre feine Schattenkontur zeigt bei dorsoventraler Durchleuchtung eine im Niveau des II. Interkostalraumes fallende leichte Einkerbung, bei ventrodorsaler Durchleuchtung. bildet sie aber einen regelrechten Bogen, mit nach auswärts gerichteter, seichter Konkavität.

Ungefähr 2 Monate nach der Brauerschen Operation stellt sich die Patientin von neuem vor. Nan ergibt sich, dass sie gravid ist, im 6. Monat der Schwangerschaft. Status praesens (4. Sept. 1912): die Frau fühlt sich bedeutend gestärkt, sie ist im häuslichen Kreise völlig arbeitsfähig; Appetit sehr gut, Stuhl regelmässig, Schlaf gut, Husten hat weiter abgenommen, Auswurf 0 , Dyspnoe 0, Nachtschweiss 0 . Sie referiert über volle Afebrilität. Aussehen geradeza wunderbar. Gewicht: $59,3 \mathrm{~kg}$ (Zunahme: 6,7 kg), nur der Puls will nicht nnter 100 sinken, er ist grösser, doch weich. 
Thoraxbefund vor der 3. Reinsufflation: Rechtgvorne: das Krönigsche Schallfeld normal breit, bei stärkerer Perkussion gibt es Schachtelton. Intensiver Schachtelton in der infraklavikulären Gegend. In der vorderen Axillarlinie reicht der Luftschall bis zur. VIII. Rippe, doch ist der Pektoralfremitus nicht abgeschwächt und man vernimmt ein zwar dem linksseitigen gegenüber abgeschwächtes rauhes Atmen. Rhonchus 0 . Rechts hinten: die obere mediale Luftschallgrenze steht höher als links, die untere Grenze etwas tiefer; besonders oben ausgesprochener Schachtelton, doch der Fremitus pectoralis ist auch lier erhalten und es ist ein intensives, ranhes Atmen hörbar. Rhonchi werden auch bier nicht gefunden.

Am 15. Sept. 3. Nachfüllung mit Einblasung von $1500 \mathrm{ccm} \mathrm{N}$, wonach die Kranke über etwas Dyspnoe klagt. Nach dieser Reinsufflation stellt sich der inzwischen etwas abgenommene Pneumothorax completus von neuem her, mit einer besonders vorne sehr mächtigen Kompression der rechten Lunge, über welcher nanmehr vorne durchwegs Schachtelton und Fehlen der Atmung herrscht und sogar hinten das Atmungsgeräusch fast völlig verschwunden ist.

Am 14. Oktober ist der Status der folgende: Bei der im 7. Graviditätsmonat befindlichen Frau besteht Eupporie und Afebrilität. Appetit gut, Husten wenig, Auswurf 0, Dyspnoe 0. Das rechte Krönig sche Schallfeld ist auch hinten auffallend breit, die Perkussion der obersten Brustwirbel gibt Luftschall (ein von Prof. Baron A. v. Korán yi für den sich auch auf die Pleurakuppel erstreckenden Pneumothorax festgestelltes, wertvolles Symptom), rechts durchwegs ausgesprochener Schachtelton, der Fremitus pectoralis abgeschwächt, bloss das Atmungsgeräusch fehlt nicht vollkommen. Alles in allem hielt sich der Pneumothorax seit der letzten Reinsufflation noch fester als zuvor, worin wir der permanent höheren Diaphragmastellung infolge des Druckes von seiten des graviden Uterus eine Rolle zusagen. - Patientin bekommt bei der 4. $\mathrm{N}$ a chfüll ung am 14. Okt. mit Rücksicht auf obigen Befund bloss $600 \mathrm{ccm}$ N. Nach dieser Einblasung hört das Atmungsgeränsch rechts vorne auf und wird rechts hinten ad maximum abgeschwächt.

Nach einem Monat, am 14. Nov., kam die briefliche Mitteilung von der Patientin, dass sie in der linken Thoraxhälfte Schmerzen verspürt, eine kleine Hämoptyse hatte und seit einiger Zeit mehr hustet.

Wir vermuten den Eintritt der gefürchteten Verschlimmerung des $\mathrm{Zu}$ standes und teilen der Patientin mit, dass sie sich je eher zeigen solle.

Am 9. Dez., zu Beginn des IX. Graviditätsmonats, stellt sich die Kranke vor. Zwar fühlt sie sich genügend kräftig (im häuslichen Kreise noch immer arbeitsfähig), zwar ist das Körpergewicht nicht unwesentlich weiter gestiegen $(64,1 \mathrm{~kg}$, jetzt gegenüber dem Originalgewicht bei der ersten Konsultation: $+11,5 \mathrm{~kg}$ ), zwar ist der Schlaf gut, Fieber wurde nicht gespürt (leider, die Patientin thermometrisierte sich derzeit nicht mehr) und der Appetit besteht auch, dennoch zeigten sich bereits gastrointestinale Störungen (Pyrose, Obstipation), der Husten vermehrte sich, Auswurf ist ziemlich viel vorhanden (,schäumig weiss", manchmal "grünlich"), und wenn die Kranke viel hustet, stellt sich Dyspnoe ein. Ihr Gesichtsausdruck ist wieder der einer Leidenden, Pulszáhl über 120 (um 130 herum). Der Pneumothorax hat sich stärker resorbiert. Obwohl das rechte Krönig sche Schallfeld noch immer breit ist und auch eine ziemliche Herzdislokation besteht, doch reicht der Luftschall rechts in der vorderen Axillarlinie nur bis zur VII. Rippe und hinten in der Angularlinie bis zur IX. Rippe, 
die Ałmang ist ziemlich gut hörbar, und die rechte Zwerchfellhälfte, deren Kuppel noch etwas abgeflacht erscheint, bewegt sich bei der Respiration vorzüglich.

Die nun ausgeführte 5 . Nachfüllung geschah bloss mit $600 \mathrm{ccm} \mathrm{N}$, da schon nach Einblasung dieser Menge des Gases ein stärkeres Druckgefühl an der Herzgegend eintrat. Nachher begibt sich die Patientin, die ihre Entbindung nach Neujahr erwartet, nach Hause mit der Verordnnng, dass sie vor dem Partus jedenfalls nach Budapest käme, damit die infolge der Entbindung insuffizient werdende Gasmenge rasch nachgefüllt werden kann.

Diesen Rat befolgte die Kranke nicht. Sie gebar in ihrer Heimat am Lande. Post partum schrieb ihr Mann auf unsere Anfrage de dato 14. Januar 1913, dass die Frau „starkes Fieber hatte, anch jetzt viel Auswurf habe und sich sehr schwach fühle". Am 25. Febr. bekamen wir auf neuerdings gestellte Anfrage vom Arzte selbst eine Nachricht, welche lautete: „Bei der Patientin ist seit der Entbindung ein rapider Verfall wahrzunehmen. Der Lungenprozess schreitet fort (dies bezieht sich nach unserer Meinung höchstwahrscheinlich auf die nicht komprimierte linke Lunge, Verff.), die Körperkräfte nehmen derart ab, dass die Kranke in 1-2 Wochen sterben werde. Es ist schade, dass die Gravidität dazwischen kam, denn der künstliche Pneumothorax war der Patientin nützlich, die Entbindung wurde aber für dieselbe leider verhängnisvoll."

Die obige ärztliche Vorhersage erfüllte sich. Die Patientin verschied am letzten Tage des Monats Februar 1913. - .

Ob nun der Verlauf ein anderer gewesen wäre, wenn die Frau sofort oder bald nach der Entbindung eine Reinsufflation bekommen hätte? - hierüber lassen sich Meinungen sagen, doch epikritisch glauben wir, dass auch eine regelrecht fortdauernde Aufrechterhaltung des künstlichen Pneumothorax die Kranke nicht mehr hätte retten können, da eben ihre Rezidive sich eigentlich nicht nach, sondern schon vor dem Partus einstellte. Der Fall war doch ein zu schwerer und für die Kompressionstherapie stricte sensu nicht mehr geeignet. 